\title{
Leading article: A possible immunochemical explanation for pregnancy associated remissions in rheumatoid arthritis?
}

Pregnancy associated remissions have been frequently observed in patients with arthritis, ${ }^{1}$ and postpartum relapses are common. Although various endocrinological and immunological explanations have been put forward, however, the mechanism responsible for these effects has yet to be established.

One possibility that has been considered is that a beneficial change in the composition of the immunoglobulins of rheumatoid patients occurs during pregnancy; and one laboratory has gone so far as to undertake a trial treatment of a group of patients with severe arthritis with a preparation of placenta eluted gammaglobulins. ${ }^{2}$ It was suggested that the clinical improvement which ensued in most $(64 \%)$ of the patients could possibly have been attributed to the polyspecific anti-HLA-DR activity of the gammaglobulin preparation on cells bearing this alloantigen. It should be recognised, however, that the relatively protracted and vigorous procedure adopted in retrieving from trophoblastic cells the 'therapeutic' IgG used in this study probably resulted in its structural alteration; one consequence of which could have been an increase in its in vivo reactivity with rheumatoid factors.

In this connection it is interesting to note that it has been assumed that the $\operatorname{IgG}$ of rheumatoid patients themselves is structurally different from that of normal individuals, ${ }^{3}$ though Normansell and I failed to obtain evidence for this from analytical ultracentrifugation studies of the interaction of autologous and isologous $\mathrm{IgG}$ with $\mathrm{IgM}$ rheumatoid factor. ${ }^{+}$Significantly, more recent studies have indicated that the composition of its oligosaccharide moieties can influence the autoantigenicity of IgG. ${ }^{5}$ Moreover, autologous IgG which has been asialylated by neuraminidase treatment has been shown experimentally to induce rheumatoid factor and arthritis in the rabbit. ${ }^{6}$ It was of particular interest, therefore, to find that a detailed analysis of the carbohydrate composition of total IgG isolated from the sera of a group of patients with rheumatoid arthritis showed a reduction in the extent of galacytosylation of the $N$-linked biantennary oligosaccharide chains, compared with that found in the IgG of normal controls. ${ }^{7}$ One possible immunochemical consequence of this may be the exposure of a putative peptide autoantigenic site, no longer protected by the 'umbrella' of the fully galacytosylated oligosaccharide chains (linked to asparagine residue 297 within the $\mathrm{CH}_{2}$ domains). Another possibility is that a reduction in the extent of galactosylation of rheumatoid IgG results in its autoaggregation; and, in this respect, it is interesting to note that self associated IgG has been found in the sera of certain rheumatoid patients. ${ }^{8}$

In light of these earlier observations, the findings now reported by Pekelharing and associates, of a temporal compensation during pregnancy of the rheumatoid arthritis associated undergalactosylation of $\mathrm{IgG}$, are of particular interest. ${ }^{9}$ It will be important, however, for these investigations to be extended to confirm that the extent of galactosylation of the $\operatorname{IgG}$ of rheumatoid patients likewise reverts to normal during pregnancy. In addition, alternative explanations of the remission seen in pregnant rheumatoid patients will need to be excluded. For instance, it is conceivable that similar changes in the carbohydrate composition of other plasma proteins occur during pregnancy, including that of $\operatorname{IgA}$, which arguably is more intimately involved than IgG in the immunopathology of rheumatoid arthritis. ${ }^{10}$ It will also be important to establish that altered hormonal status during pregnancy does not lead to other immunological consequences, besides a change in the extent of galactosylation of immunoglobulin. Sex hormones are known to influence normal and abnormal (e.g., autoimmune) immunological responses ${ }^{11}$ controlling the levels of total immunoglobulin (notably $\operatorname{IgA}$ and $\mathrm{IgG})^{12}$ and specific antibodies of these classes ${ }^{13}$ in the female reproductive tracts.

It is quite possible that the other observation of Pekelharing and associates, ${ }^{9}$ that the circulating IgG in pregnancy contains higher than normal amounts of sialic acid (besides more galactose), will turn out to be of greater clinical significance, however, because, as these investigators discuss, this change could well compensate for the decreased oligosaccharide sialylation which is likely to take place in IgG that has been synthesised by B lymphocytes infected with neuraminidase containing (e.g. influenza ${ }^{1+}$ ) virus. This would counteract the pro- 


\section{Editorial}

duction of altered $\mathrm{IgG}$, which the rabbit studies ${ }^{5}$ referred to earlier suggest would be capable of eliciting autoimmunogenic and even autoarthritogenic responses. These possibilities seem worthy of further experimental investigation, an undertaking which might well throw more light on the question of a viral aetiology for rheumatoid arthritis.

Rheumatology and Allergy

Research Unit

D R STANWORTH

University of Birmingham

\section{References}

1 Persellin R H. The effects of pregnancy on rheumatoid arthritis. Bull Rheum Dis 1976; 27: 922-7.

2 Sany J, Clot J, Borneau M, Ardary M. Immunomodulating effect of human placenta-eluted gamma globulins in rheumatoid arthritis. Arthritis Rheum 1982; 25: 17-24.

3 Johnson P M, Watkins J, Holborow E J. Antiglobulin production to altered IgG in rheumatoid arthritis. Lancet 1975; i: 611-3.

4 Normansell D E, Stanworth D R. Ultracentrifugal studies of the reactions of rheumatoid factor with native human $\gamma \mathrm{G}$ globulin. Immunology 1966; 10: 527-33.

5 Duc Dodon M, Quash G A. The antigenicity of asialylated
IgG: its relationship to rheumatoid factor. Immunology $1981 \subseteq$ 42: 401-8.

6 Galloway G, Leung A Y T, Hunneyball I M. Stanworth D R $\frac{}{4}$ The successful use of asialylated IgG as an immunogen and arthritogen in the rabbit. Immunology 1983; 49: 511-8.

7 Parekh R B, Dwek R A, Sutton B J, et al. Association of rheumatoid arthritis and primary osteoarthritis with changes ire the glycosylation pattern of total serum IgG. Nature $1985 ; 316$ 452-7.

8 Mannik M, Nardella F A. IgG rhcumatoid factors and self association of these antibodies. Clin Rheum Dis 1985; 11? 551-72.

9 Pekelharing J M, Hepp E. Kamerling J P. Gerwig G J, Leijnse B. Alterations in carbohydrate composition of serum IgG fron $P$ patients with rheumatoid arthritis and from pregnant women Ann Rheum Dis 1988; 47: 91-5.

10 Stanworth D R. IgA dysfunction in rheumatoid arthritis? Immunol Today 1985; 6: 43-5.

11 Ahmed S A. Penhale W J. Talal N. Sex hormones, immune responses, and autoimmune diseases. Mechanism of sex hor mone action. Am J Pathol 1985; 121: 531-51.

12 Wira C R, Sandoe C P. Sex steroid hormone regulation of $\operatorname{Ig} \dot{0}$ and IgG in rat uterine secretions. Nature 1977; 268: 534-5.

13 Wira C R. Sandoe C P. Specific IgA and IgG antibodies in the secretions of the femalc reproductive tract: effects of immunization and estradiol on expression of this responsc in vivo. Immunol 1987; 138: 4159-64.

14 Duc Dodon M, Cecchelli R, Cacan R. Gazzolo L. Verbert A Viral neuraminidase and cellular ectosialyltransferase in huma lymphoblastoid cells infected with influenza virus. Biochimite 1984; 66: 493-6. 